\title{
DESPARATELY SEEKING MARMOUSI: STARRING SG-PRESTACK DEPTH MIGRATION AND FOCUSING ANALYSIS
}

\author{
François Audebert \\ Compagnie Générale de Géophysique \\ 1, Rue Léon Migaux \\ Massy \\ France
}

\begin{abstract}
We took advantage of the Marmousi model to explore and check the possibilities of focusing analysis (resulting from S-G prestack depth migration, where $S$ stands for shot and $G$ for geophone), as a tool for determining a "background" velocity model. As the Marmousi model was unknown to us, we decided to use only the geological information in our possession, and the minimal processing necessary for the S-G prestack depth migration.

Regarding the pre-processing, a correction of the time-lag of the wavelet is sufficient to ensure depth calibration. Further deconvolution and multiple-removal are desirable (but not compulsory) for better legibility of focusing analyses.

Regarding determination of velocities, focusing analyses perform very well in areas of calm structure, allowing us to find depths, velocities and smooth lateral velocity gradients. In areas of complex structures, information given by focusing analysis is harder to exploit, particularly in the case of sharp lateral velocity variations : where strong deviations of rays occur, no quantitative information is to be expected, but only a qualitative check of geological interpretation.
\end{abstract}

\section{INTRODUCTION}

The Marmousi model is a synthetic model with a realistic complexity of structures and a realistic distribution of reflectors. It is thus a good benchmark for structural imaging methods. Moreover, the geologic model was unknown to us until the day of the workshop. It was thus a good case for testing the capabilities of a new method claiming to retrieve velocities from seismic data.
This method is the S-G prestack depth migration, which gives depth images, and its by-products, focusing analysis spectra and scans. The focusing analysis spectra can be seen as extensions of NMO velocity spectra and the focusing analysis scans as sorts of Velocity-scans for complex move-out cases.

The S-G method, (S for sources, $G$ for geophones) performs a downward extrapolation of the wavefield recorded at each source and each geophone position, according to the principle of the "sinking survey". Then imaging is obtained by extracting at each extrapolation depth and each horizontal location the wavefield extrapolated to zero-offset and zero-time.

The imaging ability of S-G prestack depth migration was proven by IFP at the workshop : using exact velocities, the image of the Marmousi model was as satisfactory as could be expected.

The capacity of focusing analyses to cope with complex 2D media was not ensured by theory and was the object of our study for this workshop. In order to illustrate this capacity clearly, we decided to use no other velocity analysis tools, but the log information supplied for the workshop and S-G depth migration products. Moreoever, we used those products in a pre-specified iterative process, carefully describing under which conditions and which assumptions we proceeded from one iteration to another.

This policy of using only one type of tool is quite different from the philosophy adopted by the other participants for the workshop : generally they used combinations of sophisticated methods (what is generally done in reality, as a matter of fact). 


\section{S-G PRESTACK DEPTH MIGRATION}

The S-G prestack depth migration was developed under the trademark MIGPACK by Denelle (CFP) and Jeannot (Dataid), following pionneering work by Yilmaz and Chambers.

The S-G prestack depth migration is based on the principle of the "sinking survey" : the whole prestack shot-geophone wave field is extrapolated downwards in depth alternatively along shot gathers and geophone gathers (according to the reciprocity principle, shot gathers and geophone gathers represent physical wavefields that can be processed with the wave equation). We thus obtain the S-G wavefield that would have been recorded at extrapolation depth Z, (as far as only primaries are concerned). Then the Imaging Principle is applied at depth Z, stating that the reflectivity at shot position $X$ and depth $Z$ is proportional to the pressure recorded at time $\mathrm{t}=0$ and offset $\mathrm{h}=0$. A migrated image is thus constructed step by step in $\mathrm{X}$ and $\mathrm{Z}$. If we choose the case of a flat reflector in a homogeneous medium, the extrapolation of the wavefield collapses the reflection hyperbolae on $S$ or $G$ gathers to zero offset. If extrapolation is performed with an exact velocity $\mathrm{Vr}$, the reflection hyperbolae will collapse to their apex and will be totally "focused" at time zero and offset zero for an extrapolation depth $\mathrm{Zr}$ equal to the real depth of the reflector. The image of the reflector has then maximum amplitude. On the other hand, if the extrapolation is performed with a migration velocity $\mathrm{Vm}$, different from $\mathrm{Vr}$, with for instance $\mathrm{Vm}>\mathrm{Vr}$, then focusing will occur before imaging; the hyperbola will still collapse at zero offset but at a time not equal to zero, and for an extrapolation depth Zf (focusing depth) different from real depth $\mathrm{Zr}$ and imaging depth $\mathrm{Zm}$. In the imaging stage, for extrapolation depth $\mathrm{Zm}$, the hyperbola will be defocused and energy extracted at time 0 , offset 0 , will not be maximal.

We have for imaging :

$$
\frac{\mathrm{Zm}}{\mathrm{Vm}}=\frac{\mathrm{Zr}}{\mathrm{Vr}}
$$

For focusing :

$$
\mathrm{Zf} * \mathrm{Vm}=\mathrm{Zr} * \mathrm{Vr}
$$

the combination of these formulae gives $\mathrm{Zr}$ :

$$
\mathrm{Zr}=\sqrt{\mathrm{Zr} * \mathrm{Zm}}
$$

which is approximated by :

$$
\mathrm{Zr}=(\mathrm{Zf}+\mathrm{Zm}) / 2
$$

then an estimate of $\mathrm{Vr}$ is deduced :

$$
\mathrm{Vr}=\mathrm{Vm} * \mathrm{Zr} / \mathrm{Zm} \text {. }
$$

Thus with $\mathrm{Vm}$ and $\mathrm{Zm}$ being known, we only need a measure of $\mathrm{Zf}$ to obtain an estimate of both $\mathrm{Zr}$ and $\mathrm{Vr}$.

In order to obtain focusing analyses, we store pressure at offset zero, for all times, for all extrapolation depths, and of course for all horizontal locations. The focusing data volume thus obtained can be displayed in several ways.

One is called the focusing analysis (spectra) and is analogous to standard velocity analysis spectra. Energy at each surface location is displayed as a function of $\mathrm{Zm}$ and depth error $E=(\mathrm{Zf}-\mathrm{Zm}) / 2$. This gives a local, precise estimate of $\mathrm{Zf}$, but seismic character and lateral continuity are lost.

The second display could be called Z-scan as it is analogous to (NMO) velocity scans. The pressure amplitude, for one depth error $E$ specified, is displayed as a function of $X$ and $(Z M+E)$. A $Z$-scan is a locally over or under migrated analogue of the migrated section (it is the migrated image if $E=0$ ). It can be understood as an image obtained by applying the imaging principle for a time different from 0 . It is only a display of the focusing data volume along other axes than the ones used for focusing analysis spectra. This Z-scan display gives a global view and preserves the seismic character of the focusing data.

But remember that the focusing formula

$$
\mathrm{Zf} * \mathrm{Vm}=\mathrm{Zr} * \mathrm{Vr}
$$

relies on the following hypotheses :

- Vm close to $\mathrm{Vr}$, but if $\mathrm{Vm}$ is very different from $\mathrm{Vr}$, the iterative process can be expected to converge properly provided the next hypothesis is fulfilled.

- Horizontally stratified, laterally invariant velocity medium. This hypothesis is quite critical. How to proceed outside this ideal case is exactly what we are trying to find out.

The focusing formula can be extended from homogeneous to horizontally stratified media. Noting that if velocities are perfectly known down to the last but one layer, determining the velocity and depth of the last layer is a homogeneous case problem. As a consequence, the method fully applies in a layer-by-layer stripping process. In fact, we can even encompass several layers at a time : the iterative process converges very fast, as long as we remain within the horizontally-stratified medium assumption. 


\section{PRE - PROCESSING}

S-G prestack depth migration might allow for rather basic preprocessing. The proof is that IFP showed a good depth image with unprocessed (prestack) data. But it was noise-free synthetic data. Since we were not provided with the source signature, and since this signature was not easy to extract from the seismic data, the fact is that we performed some iterations with no pre-processing but with muting of the refractions. This gave satisfactory results, see iteration 0 to 3, Fig. 1 and 2.

Nonetheless, although the recovery of the timelag of the wavelet alone is sufficient to ensure a good depth calibration with respect to the surface, deconvolution is recommended for the quality of the migrated image and the resolution of the focusing spectra.

Water bottom multiples can be recognised on focusing spectra. We can manage with them in Marmousi, but still it is preferable to get rid of them to avoid interferences with primaries and ensure a better legibility of focusing spectra and scans.

Amplitude calibration was automatically ensured in the Marmousi case. S-G migration works with the assumption that all sources and receivers are identical. Since real data do not always fulfill this requirement, we then have to make the necessary amplitude calibration.

Nothing else is necessary at the pre-processing stage, but all the methods aiming at retrieving velocity information are welcome to complement to $S$ $G$ prestack depth migration.

\section{RETRIEVING THE MARMOUSI MODEL}

Using S-G prestack depth migration, we followed this scheme :

Iteration 0 :

Migration with a constant gradient, 1D model.

Iteration 1 :

Introducing geological information.

Iteration 2 :

Taking into account focusing analyses where they are readily interpretable and justified.

\section{Iteration 3 :}

Taking into account focusing analysis information where it is still relevant.

Iterations 4 and more :

Performing iterations with careful and cautious interpretation of non obvious focusing analyses.

\section{Introduction of geologic information}

The available geological information consisted of two well logs located just outside the section and in a geological description. I must admit I misunderstood this "geological" description and had little confidence in it. One reason is that the structure to be found was beyond my geological background (a proof that geologists should participate in the process, and that prestack depth migration is both a processing and an interpretative tool). Another reason is that the geology described was not consistent with the (geophysics from the) data.

For instance, the growth faults mentioned were obviously in the wrong direction according to seismic data. So the geological description could not be considered as correct or reliable, and those faults did not seem to be standard growth faults but some kind of tectonic ones, resulting from upward push of salt for instance.

On the basis of the observed data, I followed this latter geologic hypothesis, but it was not the correct one and it trapped me in a incorrect geologic model.

After the geological description, I proceeded to analyze the well logs : I was not puzzled by the permutation of logs, their location was obvious from the seismic data. I used them with confidence. In a first step, I defined a constant vertical regional velocity gradient (from these well logs), and then performed an initial iteration of migration to obtain the first focusing analyses, iteration 0, Fig. 1-a.

After this iteration, I used focusing analyses along with the well logs and the migrated section to identify significant reflectors, i.e. significant velocity domain boundaries. From the focusing analyses and the migrated section, I selected energetic reflectors or reflectors marking a significant change in velocity, and picked them on the migrated section to define their lateral extent, thus enclosing velocity domains.

Then I tried to identify these velocity domains on the migrated section with velocity intervals on the well logs. When it was possible, I assigned to the whole domain the velocity from the well log : a sort of lateral extrapolation of the velocities from the well logs. In the central area where such an extrapolation was not possible, I put some average regional velocity functions, a rough interpolation between the averaged well logs.

This produced iteration 1, Fig. 1-b. 


\section{Taking into account fully justified focusing analyses}

After iteration 1, fig. 1-b, I extracted velocity information from focusing analyses but only where it was readily justified : this means areas where the horizontally stratified medium assumption is met, strictly or allowing for very gentle dip, with the remark that this assumption can be met locally at some singular points : tops of anticlines, bottom of synclines and diffractors with a $1 D$ velocity medium in a certain "cone" over these points.

In order to build a new velocity model, we work with five tools :

- a zero offset or stack section, this is a time display that is unaffected or little biased by the velocity we chose. It is a useful and reliable reminder of the appearance of the seismic data we have ;

- the previous velocity model,

- the resulting migrated section,

- the focusing analysis spectra obtained,

- and the focusing analysis "Z-scans" also obtained as a by-product of the former migration.

Now the updating of the velocity model follows this scheme :

- on the migrated section we correct or pick the boundaries of the velocity domains,

- on the focusing analyses we pick depth errors preferably in the vicinity of domain boundaries,

- the migrated section and the Z-scans give qualitative information about the seismic character and the spatial behaviour of the events appearing on the focusing spectra. Multiples or spurious events can be identified in this way and differenciated from primaries.

Once we are satisfied with our interpretation of focusing spectra and selection of horizons, we can compute a new velocity model, from top to bottom.

- we begin with the upper velocity domains,

- for each domain, the interpretation of focusing spectra gives local estimates of depth error at some specific X locations. Since the new velocity domain has been picked on the previous migrated section, the new domain is defined in terms of old depths and old velocities (depths and velocities according to the "old" previous velocity model), that is to say the new domain is fully defined in terms of vertical travel time "thickness" and position. If we make the assumption that vertical travel time is invariant between the old and new velocity model, (this is true in a horizontally stratified medium, and approximate in a dipping medium), after correcting the depth with the measured depth error, we can then find a new velocity preserving the vertical travel time.
We now have a set of "new local velocities", which we interpret in terms of a single function for the whole domain. One constant vertical gradient is used over all the domain, with respect to some reference velocity at the surface, which may vary laterally in a linear or quadratic way.

We now have one velocity function for our domain, the vertical-time converted geometry of our domain being kept invariant, we deduce the final "new" depth geometry.

The scheme has to be followed from top to bottom, because for each domain we have to know beforehand the "new" depth of the top of the domain, which is nothing more than the "new" depth of the wall of the overlying domain.

The migration with this "new" updated velocity model yielded iteration 2 , Fig. 2-a.

\section{Taking into account scarcely justified focusing analyses}

We now have a fairly good image of the outer structure.

Now that we have interpreted readily justified focusing analyses, we can stretch a little further the theoretical justifications. It seems fairly relevant to state that focusing analyses would yield still good (if not precise) information about velocities in cases being not too far away from the horizontal reflector in the ID velocity medium basic assumption.

These cases correspond to :

- dipping reflectors in a homogeneous medium or with vertical velocity gradient,

- dipping reflectors in a monoclinal medium, in fact dipping reflectors with little or no distortion of the rays downwards to and upwards from the reflector,

- horizontal reflectors under a roughly 1D medium. In this case, it is expected that the distortion of the rays will statistically compensate over the offset during migration, and that migration will yield a rough estimate of an "average" velocity down to the reflector.

In all those cases, the velocity estimates given by focusing analyses, if not exact, are expected to converge in an iterative process.

We thus obtained iteration 3, Fig. 2-b.

After iteration 3, we have no more direct quantitative information to extract from focusing analyses. Iteration 3 is the farthest we went with only objective geophysical information and no interpretative geological considerations. Thus beyond that point, the information extracted from focusing analyses is qualitative and interpretative. That means that from now on we will need a geological model, some geological hypotheses and that the results of migration will be biased by the relevance or irrelevance of our geologic interpretation. 
It is interesting to observe, a posteriori, that our iteration 3, not biased by subjective interpretation, did the best in imaging the real deep structure. It is reasonably easy for a geologist looking for a "turtle shell" to see it on the section.

It is clear that in real life the participation of a geologist familiar with the area would be helpful.

With the reservation that from iteration 4 onwards the geological guideline is predominant, I tried to go ahead with focusing analyses and Z-scans, observing that except for all the cases already mentioned, no quantitative information could be extracted, and could not even be expected to converge iteratively.

\section{Off through the night}

My idea was that in the Marmousi case, the data was perfectly deterministic, with no noise or 3D effects, so that all patterns appearing on focusing spectra or Z-scans had good geophysical or numerical reasons for being there. The numerical causes have to be traced back to the finite differences algorithm we used. The geophysical causes? Well, we have to understand them. By dint of struggling with focusing spectra, I managed to qualitatively relate some typical patterns to their typical cause :

- flat reflector, dipping reflector,

- diffracting point, diffracting edge of a reflector,

- water bottom multiples,

with the reservation that in real cases, noise blurs focusing spectra.

Anyway many focusing patterns remained a mystery for me. I suspect some twice-bouncing rays as described by de Bazelaire at the EAEG, and some internal multiples, but I could not characterise them. It seems to me too, that close apart reverberating reflectors create misleading features.

When I was confronted with the real model, I discovered $\mathrm{I}$ had been right in my basic pattern recognition, and I had correctly identified many individual pieces of the jigsaw puzzle. But I failed in putting so many pieces together at their right place in a coherent geologic structure.

You can see the final result on figure 3. We can notice that the image has been further improved in the upper and outer parts of the section, and the positioning of the faults is reasonably good. But the image of the deep, central part is worse than after iteration 3 .

So what went wrong?

First, I was trapped in a wrong geologic model looking for protruding salt instead of a "turtle shell".

Second, I asked too much from the focusing analyses in a complex area, where reflection geometry is critical.
In this area, a complete understanding and determination of the geometry is a prerequisite for any, if any, quantitative exploitation of focusing data. However, even a qualitative interpretation of those data may fail to allow positioning of domain boundaries with sufficient (horizontal) precision.

It is clear that in the central area between and under the faults, a good positioning of the faults is a necessary (but may be not sufficient) condition to allow for some reliable use of focusing analyses there. It might well be a problem of the kind : the truth can be found only if the truth is known ..

\section{How did focusing analyses perform ?}

Figures 4 to 6 show the velocity logs we found (thin lines) superimposed on the "real" velocity logs (thick line) used in modelling the data, at some regular X location.

It is reassuring to note how close we came to reality in quiet areas : locations $X=4000$ and $X=$ 8000 , as well as upper part of location $X=7000$. The upper parts of logs $X=5000$ and $X=6000$, in disturbed but superficial areas, still show acceptable results. Except for the $\log$ at location $X=6000$, hopelessly wrong, the deeper sections of the logs at $X=5000$ and $X=7000$ (in disturbed areas) reveal that significant errors on interval velocities resulted in rather minor errors on the depth of the main reflectors. That is to say that it is the average velocity down to the reflector which is accurately given by the focusing analyses, rather than any interval velocity. This is not unlike the case of the stacking velocities and the well-known instability of the Dix formula. So the good news is that errors on interval velocities do not cumulate but tend to compensate over depth.

Now look at the focusing analyses, Figures 7 to 12 , obtained at the same location as the previous velocity logs. For each shot point location, there is from left to right the focusing analysis obtained after iteration 0 (constant velocity gradient model), after our final iteration and after migration with the exact model. The observations we did over the velocity logs are confirmed here:

- In quiet areas, location $3000,7000,8000$, we improved dramatically the focusing spectra between first and last iteration, and the final spectra show no significant differences with respect to reference.

- In disturbed areas, location 5000 and 6000, some improvement were obtained in the upper parts, but not in the middle and deeper parts. Not only do the spectra we obtained bear little or no similarity with reference ones, they even deteriorate in some places from first to last iteration.

- An intriguing and interesting case is location 4000 : though the estimated velocity log does not seem nonsensical compared to the reference one, the final focusing analysis just does. 
It is rather discomforting to notice that our first focusing analysis spectrum was in fact more akin to the real one than any others.

It is rather discomforting because the image we obtained was improved in this area between first and last iteration. It is surprising because we could have expected a rugged, non-exact velocity log to be a better approximation to a rugged exact log than a smooth inexact one. I am not sure this can be an argument in favour of smooth velocity model, since the point here is that we are in presence of dipping velocity contrasts, causing strong lateral deviation of rays. In such a case, a slight perturbation with respect to the exact model, either in depth or velocity, might cause a disproportionate mismatch between real and retropropagated ray, thus yielding whimsical focusing spectra. The debate is in fact : what is closer to exact, a non-exact rugged model or a non-exact smooth model, given that in this specific case a smooth model cannot be exact?

Another point we can suspect at seeing the spectra at this location 4000 : we mentioned that the errors on interval velocities do not directly add up into an error on average velocities (in other words: the errors on the thickness of the layers do not add up into an error on the depth of the layers), but we can expect the accumulation of these local errors to have a pernicious effect on the quality of the focusing analysis spectra. This can be expected because the theory states that a correct depth is fond if a correct average slowness is found, and a good focusing is found if a correct average velocity is found. So an optimal focusing at correct depth requires a good estimate of all intermediate velocities.

Finally let's have a look at the reference focusing spectra obtained with the exact velocity model : we can notice that they are :

- better than any one we obtained,

- "good" spectra we would have been very satisfied with, had we obtained them,

- nevertheless not as outstandingly "perfect" as we might have expected.

\section{CONCLUSION}

\section{S-G prestack depth migration}

A migration performed by IFP with the exact velocity model proved the full efficiency of S-G prestack depth migration in giving an accurate subsurface image of the Marmousi model, within the one-way operator limitations.

\section{Focusing analysis spectra and scans}

This case proved the full validity of quantitative information given by focusing analysis as long as strong distortions of seismic rays are not involved.
In areas where sharp horizontal velocity variations create major ray deviations, a conservative approach must be adopted :

- using focusing analysis as a qualitative and interpretative tool,

- favouring determination of geometry with respect to determination of velocity values.

On the other hand, focusing analysis seems to be a good quality control tool, which in my opinion, was not trivial. After a migration with the exact model focusing analysis spectra show a good alignment of energy on the imaging line, even in complex areas.

So, intuitively, we could say that focusing analyses, in the present state of the art, give quantitative velocity information when the migration velocity model is "not too far" from the exact velocity model. "Not too far" can be understood as "even pretty far" when the 1D assumption is fulfilled. When the real velocity model departs strongly from 1D, "not too far" might as well be understood as "infinitely close".

\section{The Marmousi model}

The Marmousi model is rich with sharp lateral velocity contrasts, faults and high-velocity wedges, all features creating strong ray deviations that no method presented at the workshop could sucessfully control. It is no coincidence that the most successful participant had precisely given most care in identifying those strong velocity singularities. This goes against the conventional wisdom that a "smooth velocity model" should be used for depth migration. Except for the open question of what a "smooth velocity model" is, especially in the Marmousi case, I cannot see how a migration velocity model could explain sharp singularities where they should be ...

\section{BIBLIOGRAPHY}

Yilmaz, O., and Chambers, R. : "Migration Velocity Analysis by Wave-Field Extrapolation" Geophysics 49

Faye, J.P., Jeannot, J.P., and Denelle, E., 1986 : "Prestack Migration Velocities from Focusing Depth Analysis" - SEG Houston

Denelle, E., Trézéguet, D., Tarantola, A., 1987 : "Non Linear Inversion by Depth Extrapolation in the SG domain" - SEG New Orleans

Julien, F., Vujasinovic, Y., Raoult, J.J., 1989 : "Continuous Velocity Analysis Based on Prestack Migration" - EAEG Berlin 
MARMOUSI ITER O

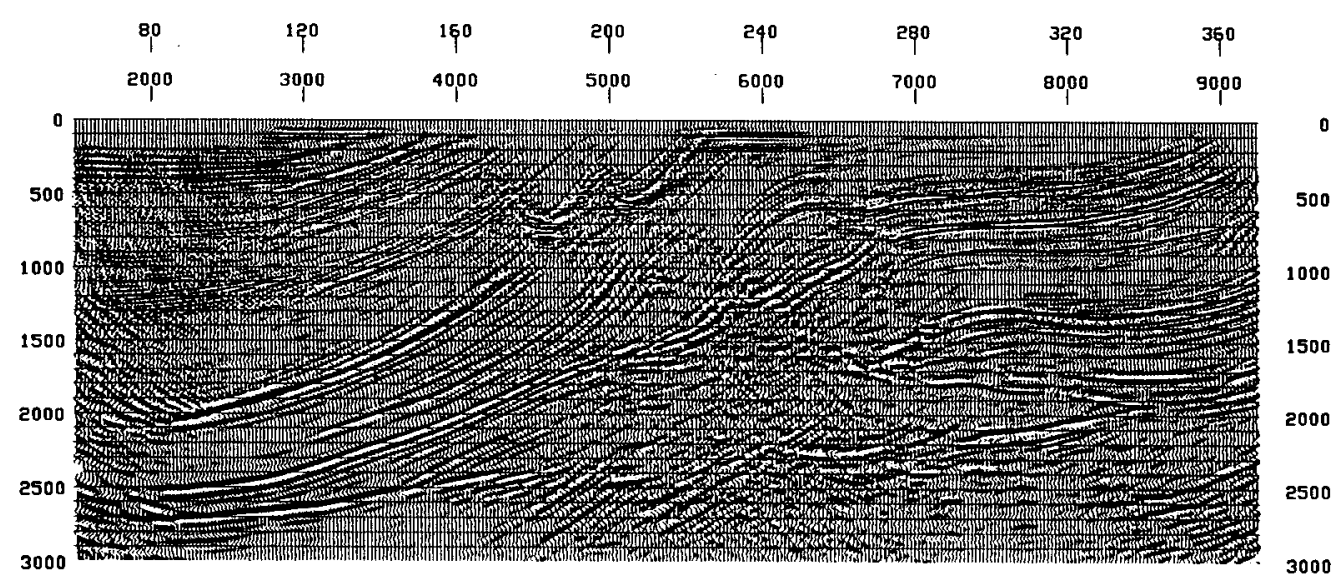

\section{MARMOUSI ITER I}

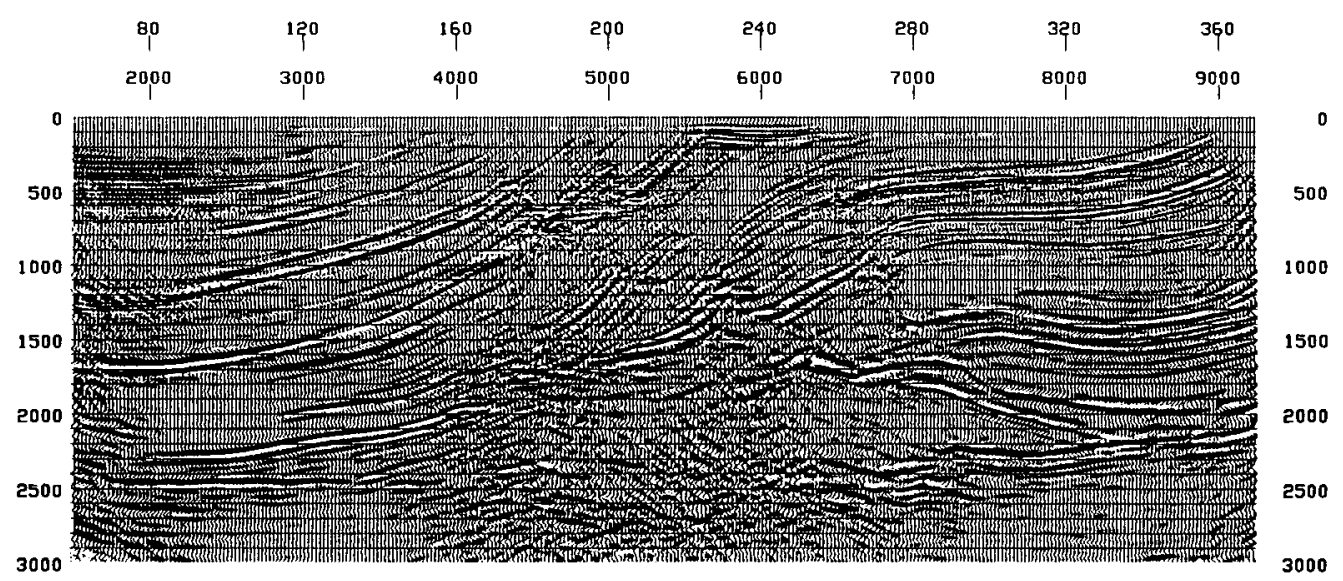

Fig. 1 - Marmousi migrated sections

a) after iteration 0

b) after iteration 1 


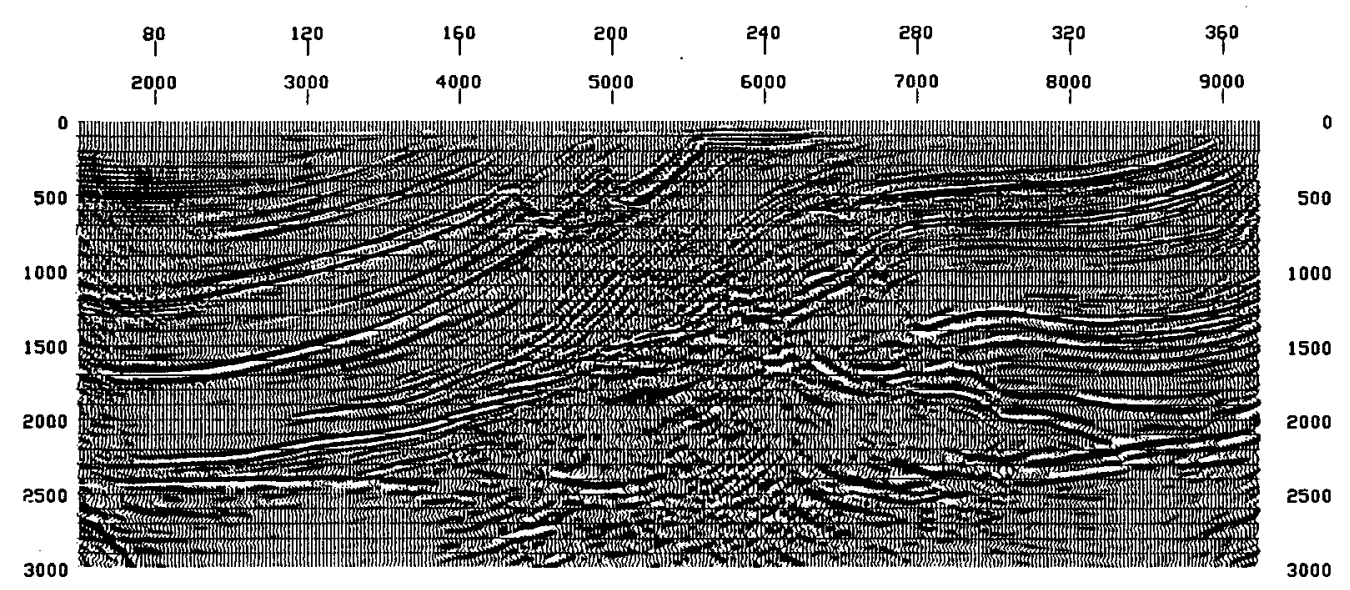

MARMOUSI ITER 3

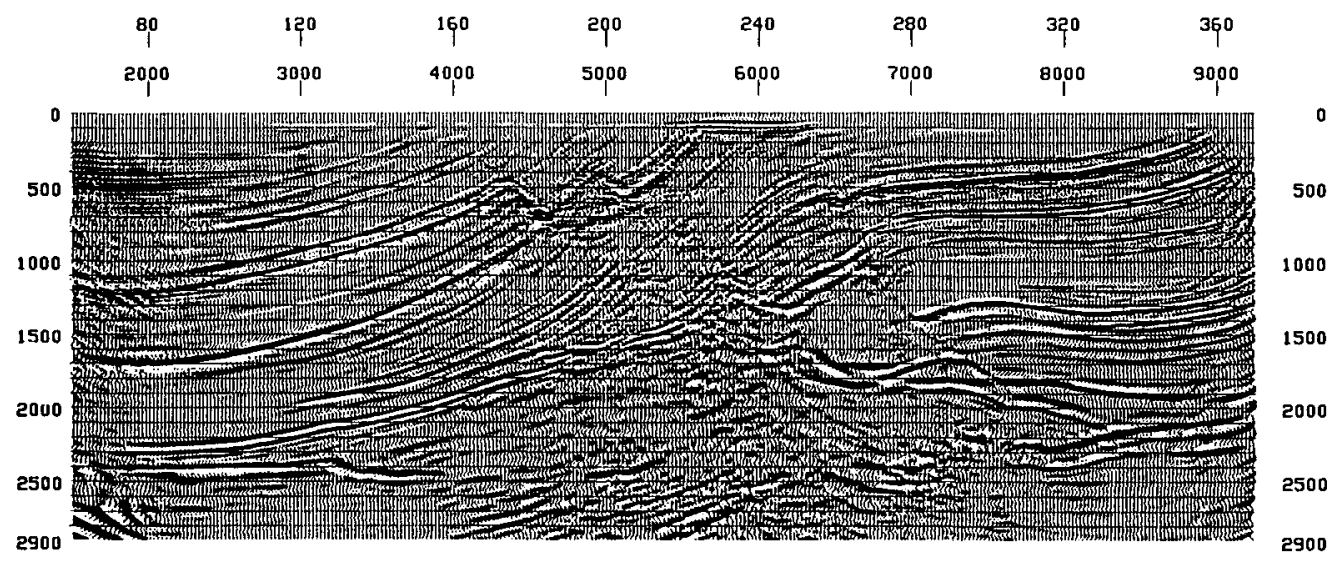

Fig. 2 - Marmousi migrated sections

a) after iteration 2

b) after iteration 3 


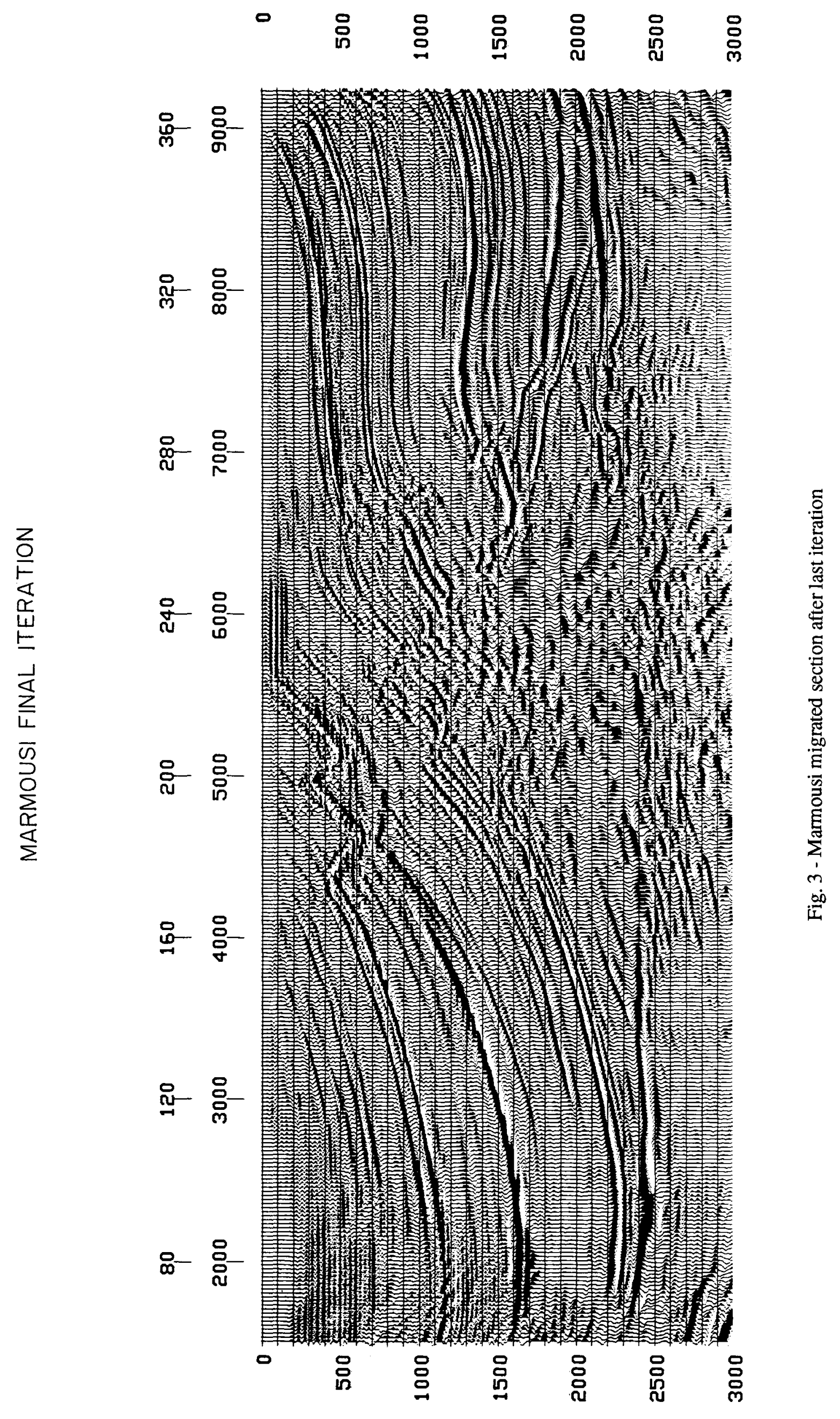



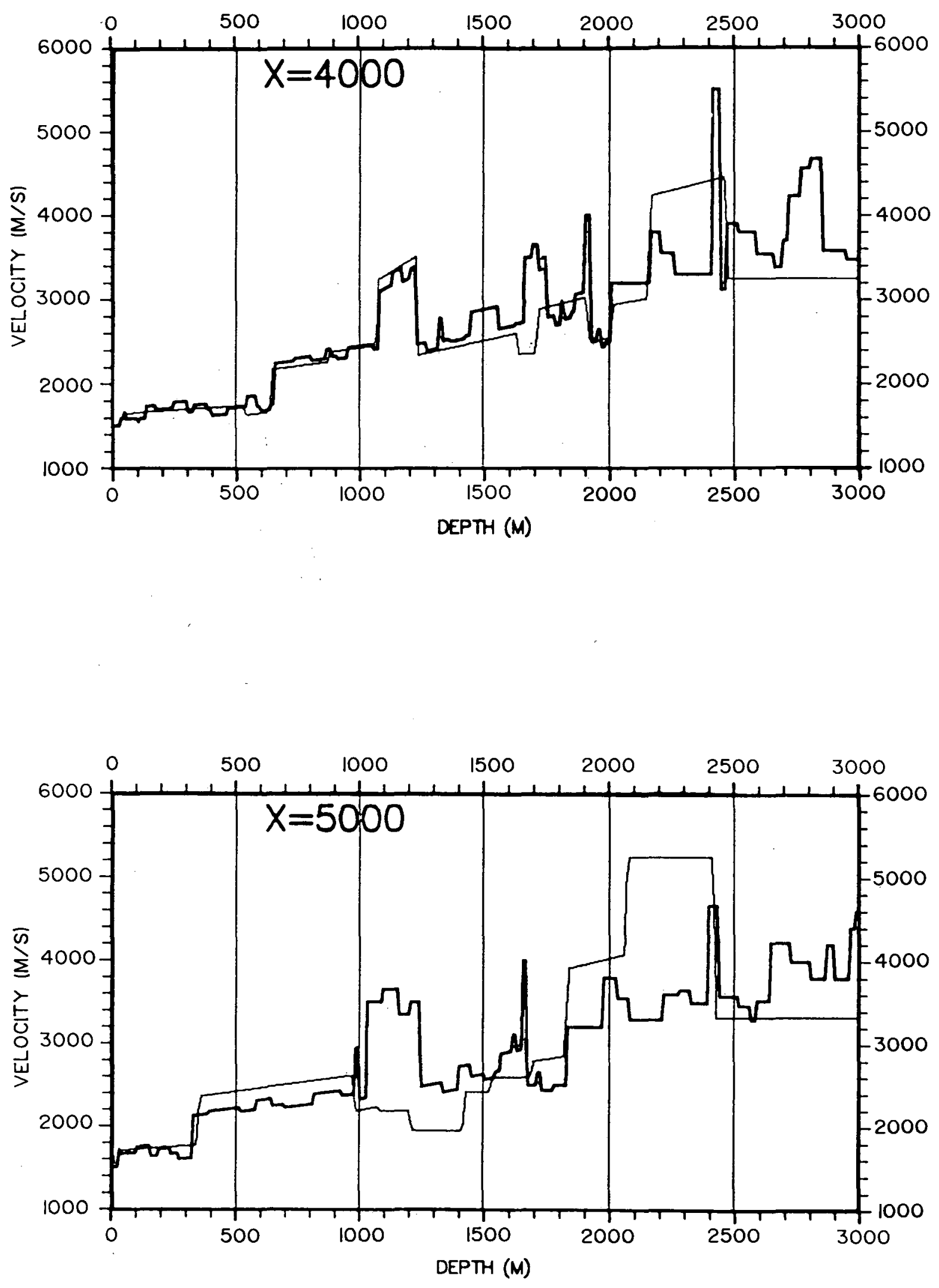

Fig. 4 - Velocity logs after last iteration (thin line) and exact (thick line)

a) $S P 160, X=4000 \mathrm{~m}$

b) SP 200, $X=5000 \mathrm{~m}$ 

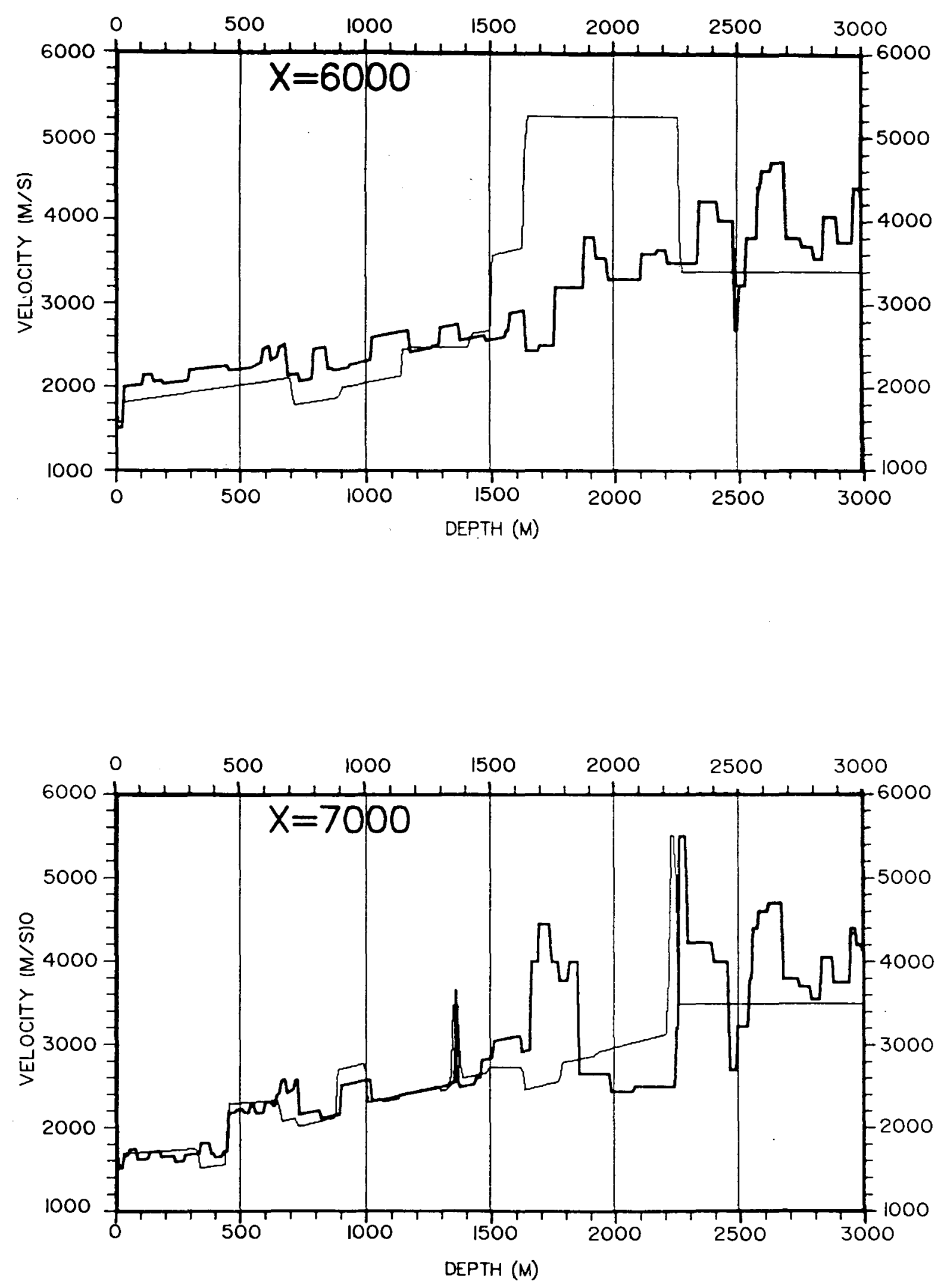

Fig. 5 - Velocity logs after last iteration (thin line) and exact (thick line)

a) SP 240, $X=6000 \mathrm{~m}$

b) SP $280, X=7000 \mathrm{~m}$ 

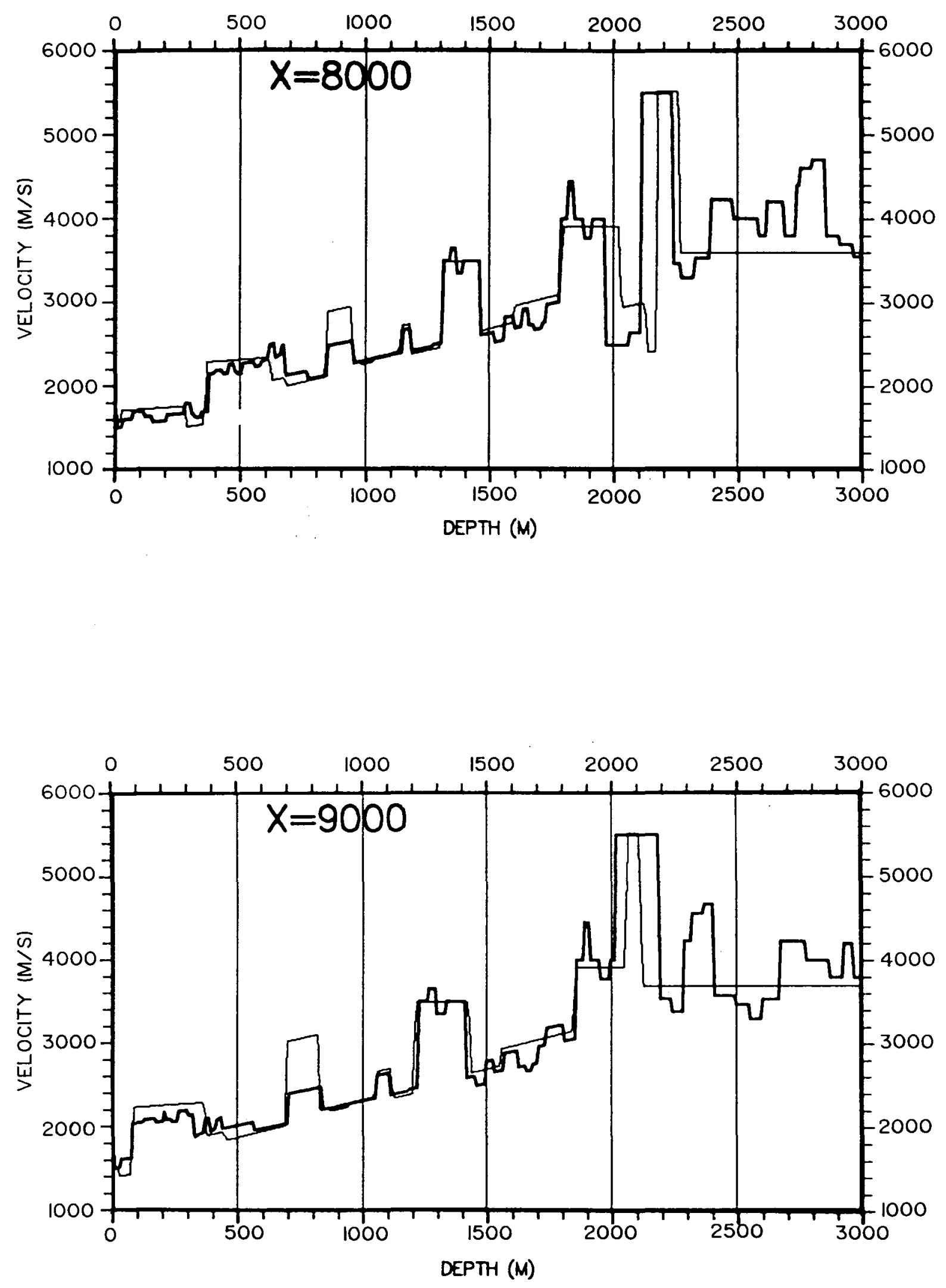

Fig. 6 - Velocity logs after last iteration (thin line) and exact (thick line)

a) SP $320, X=8000 \mathrm{~m}$

b) SP $360, X=9000 \mathrm{~m}$ 


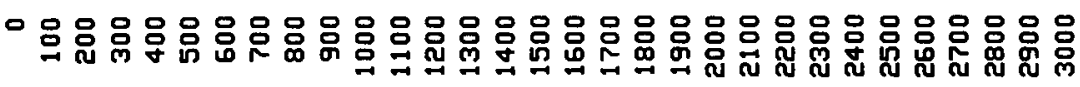

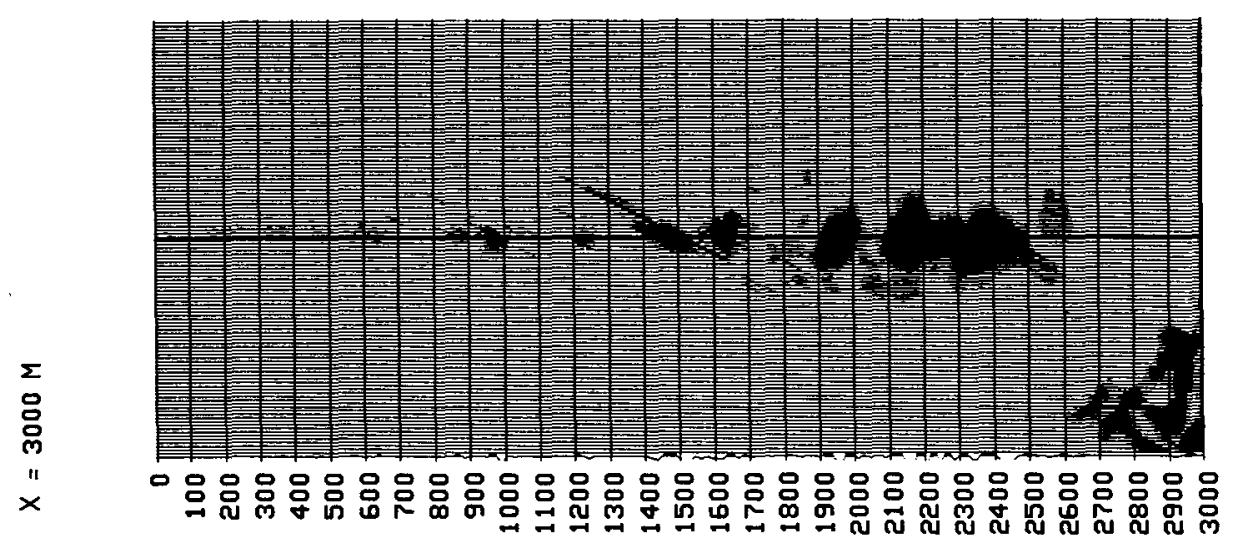

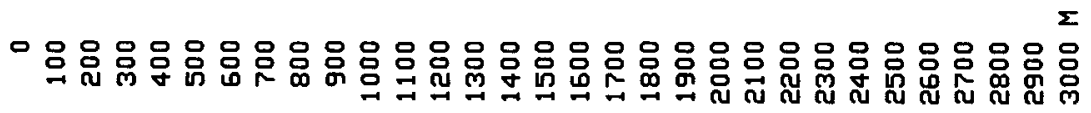
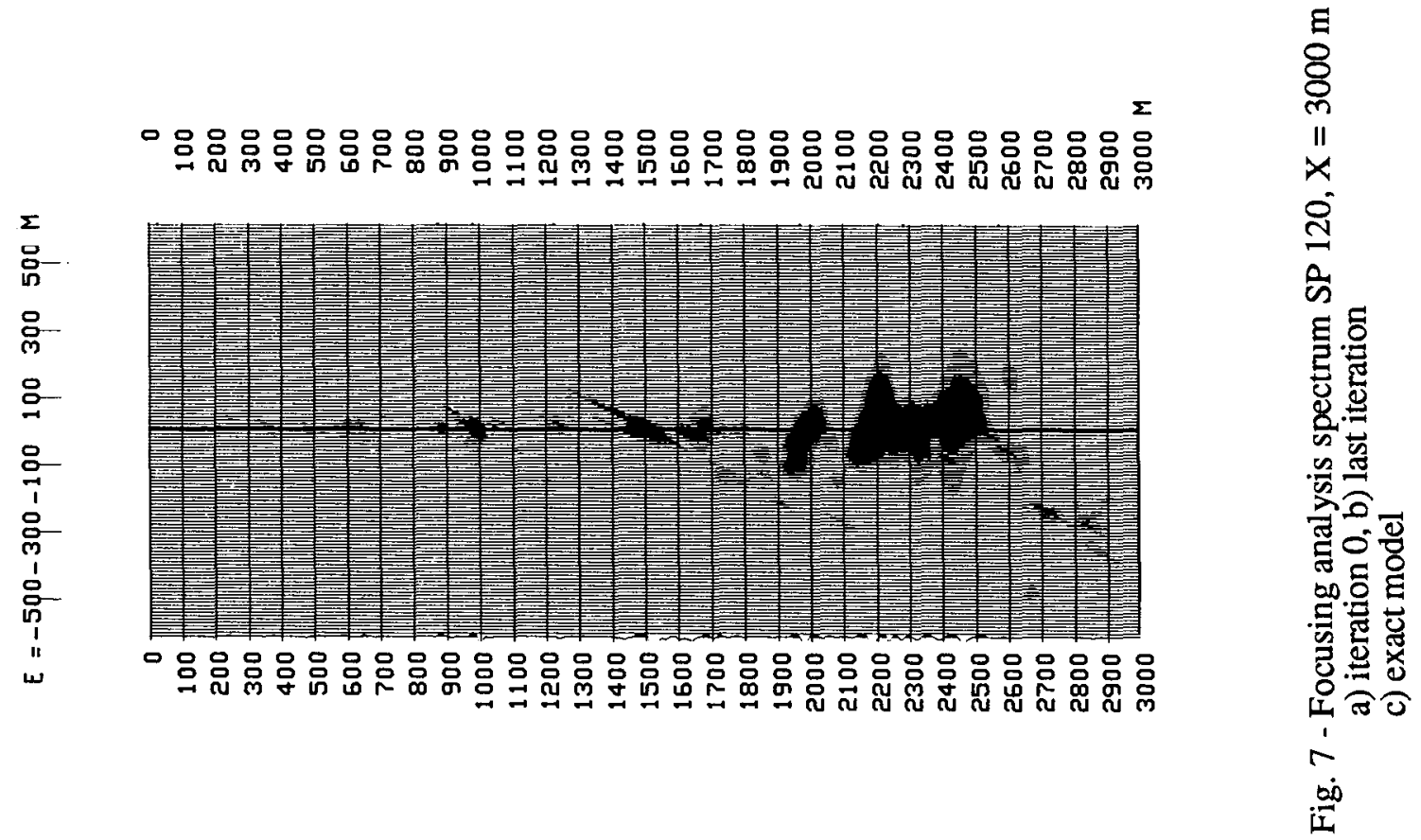

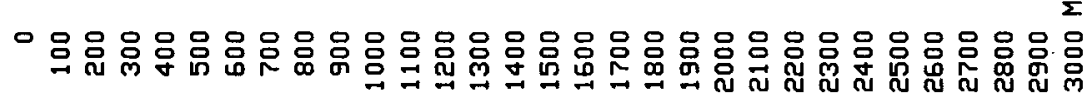

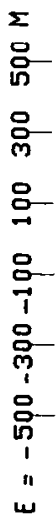

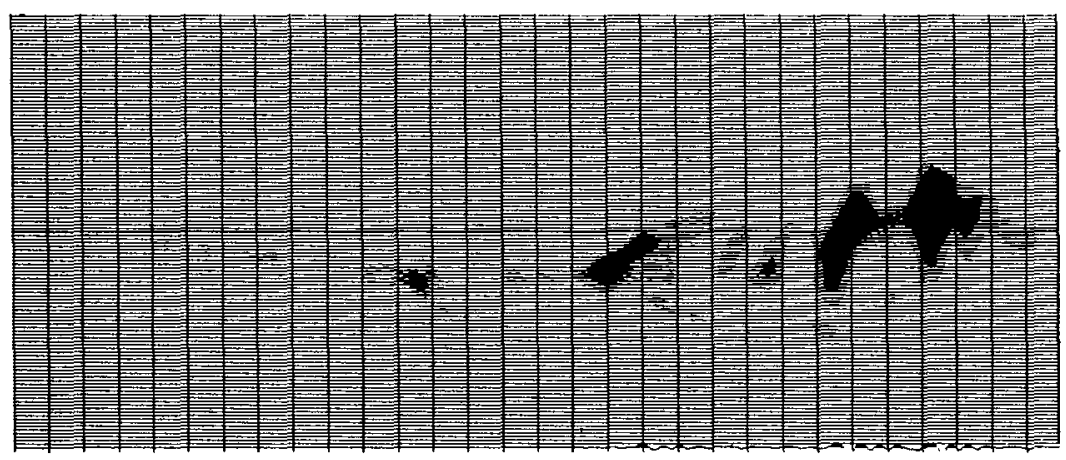

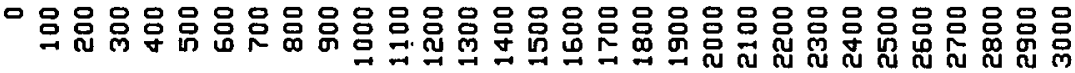




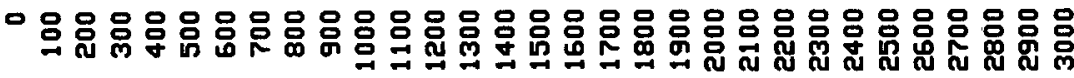

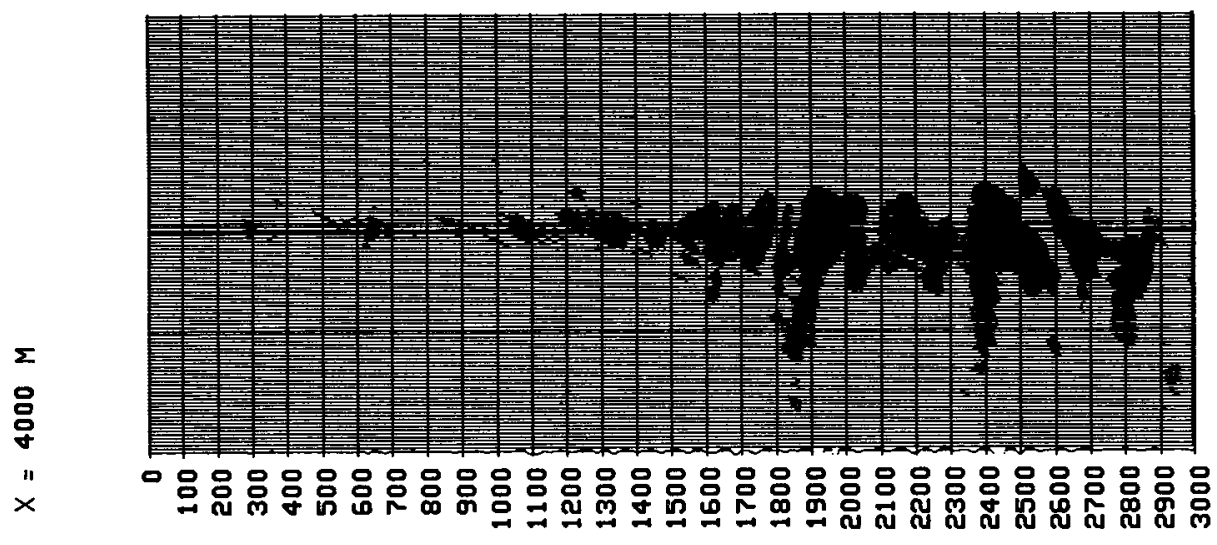

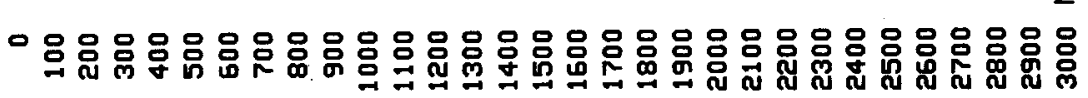

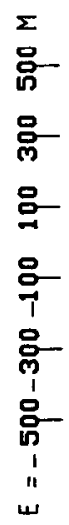

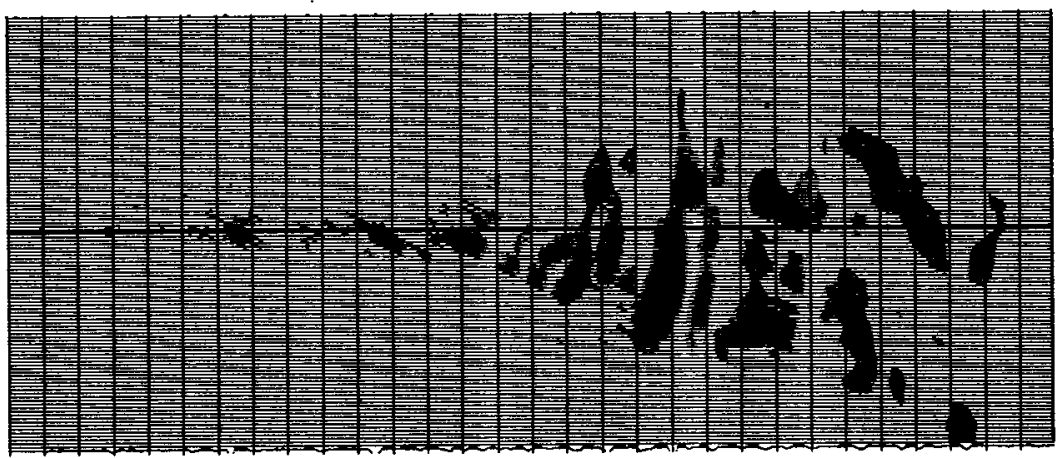

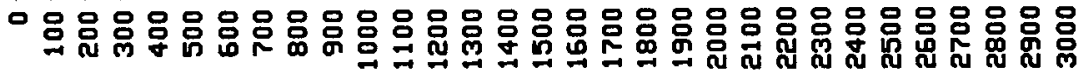

$\stackrel{8}{8}$

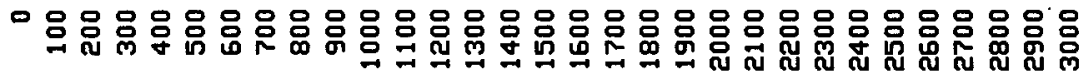

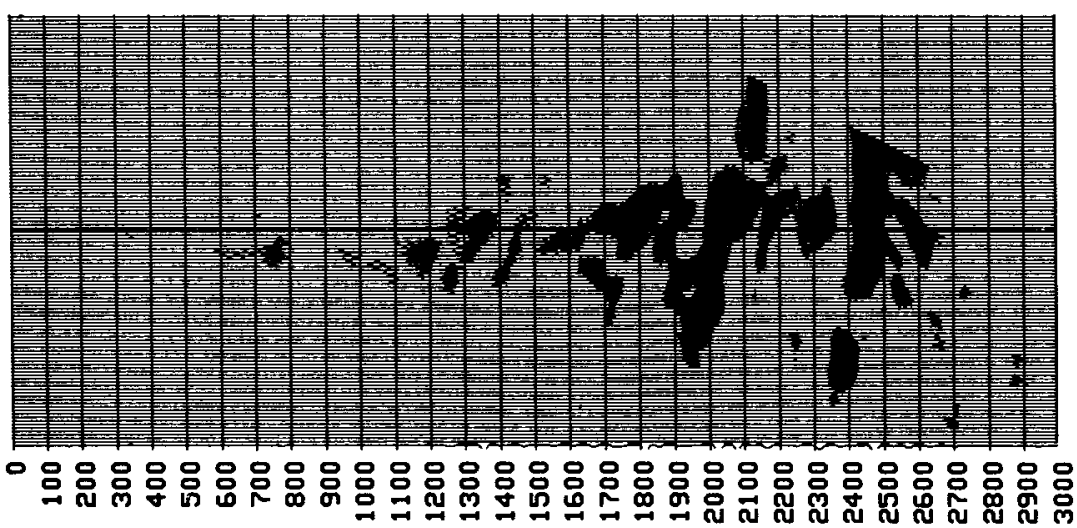




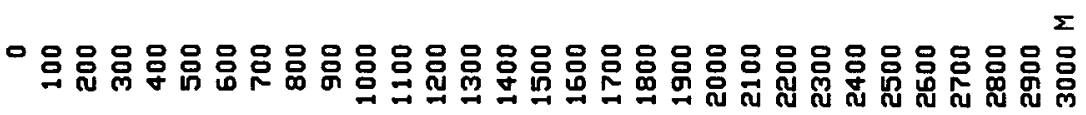

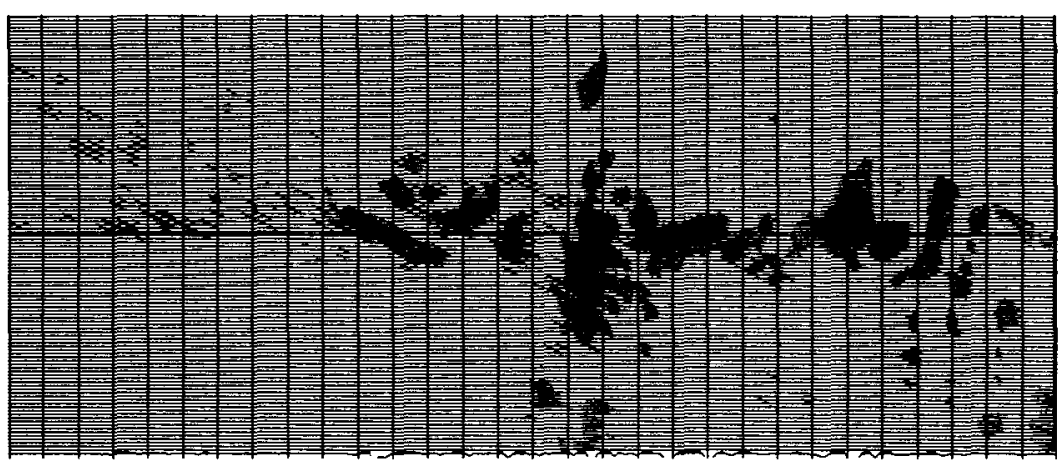

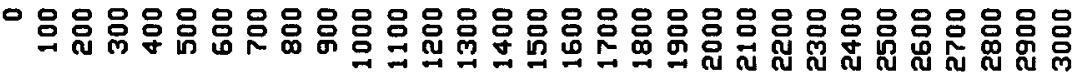

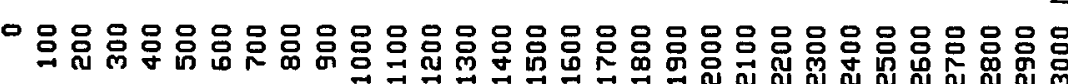
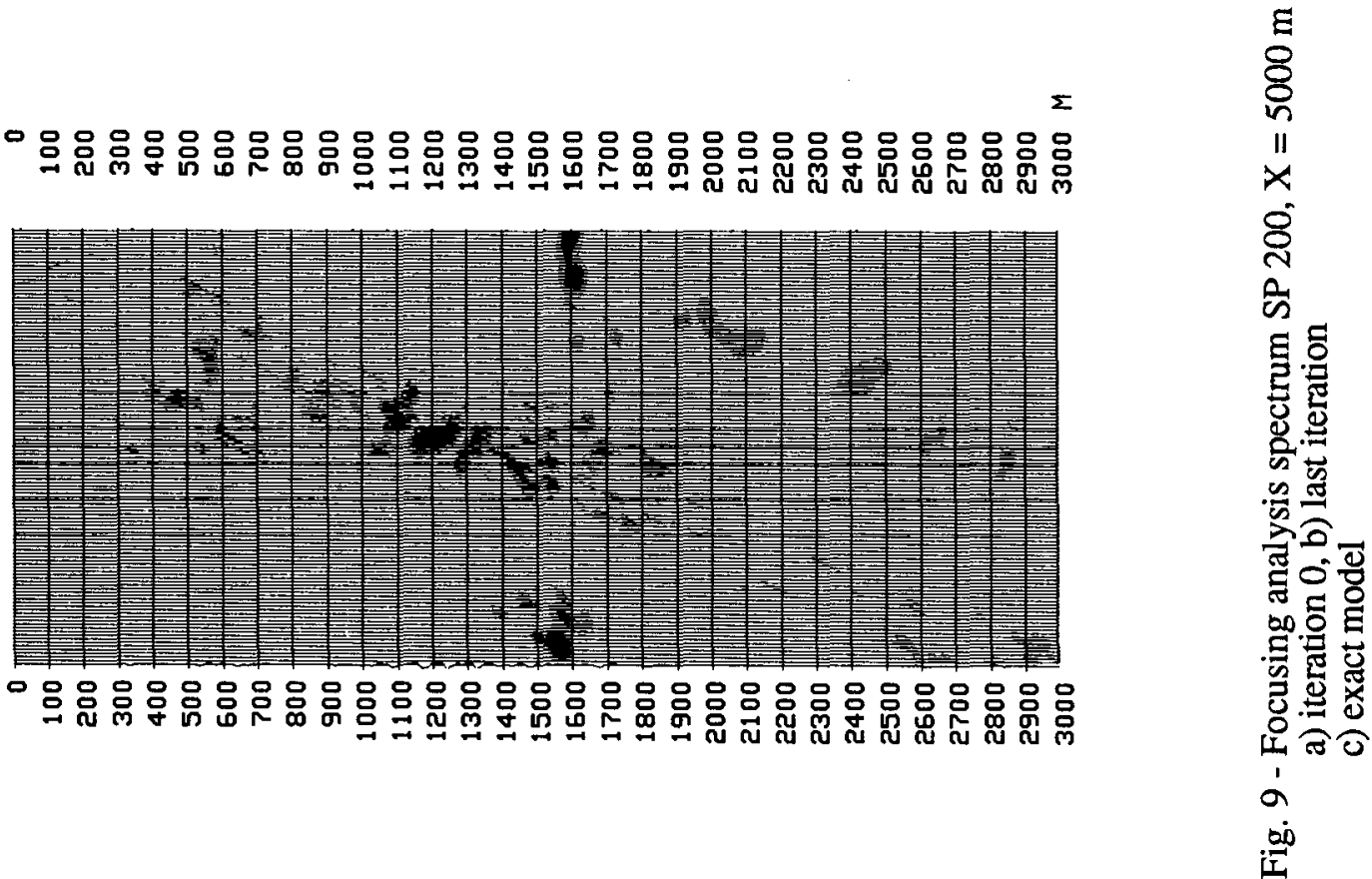

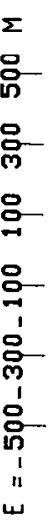

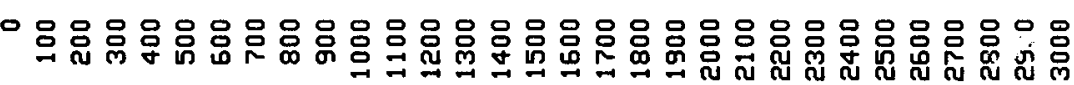

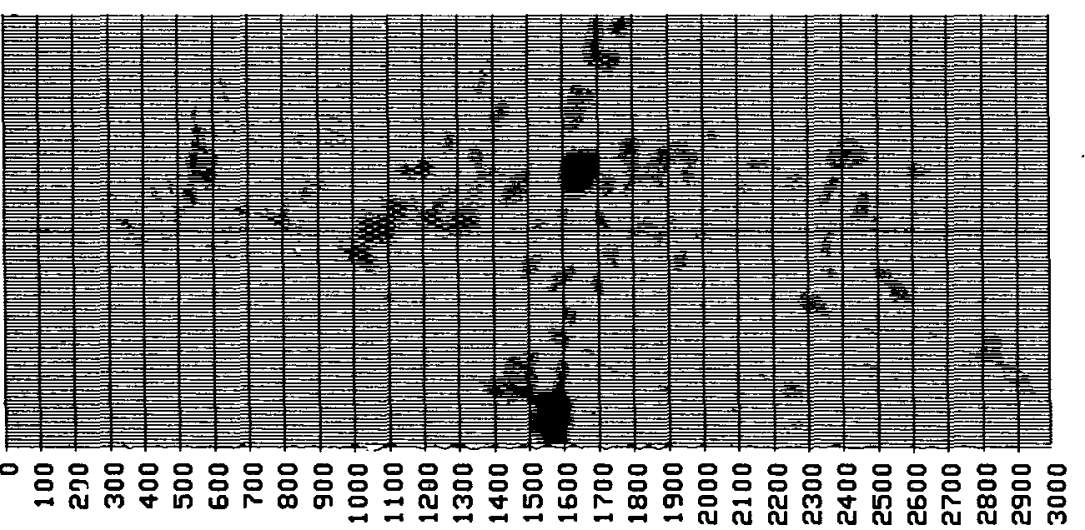




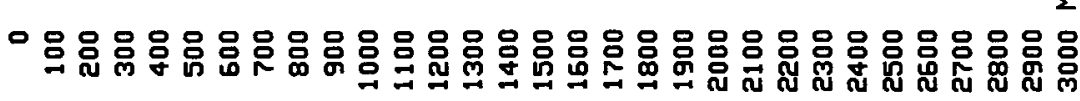

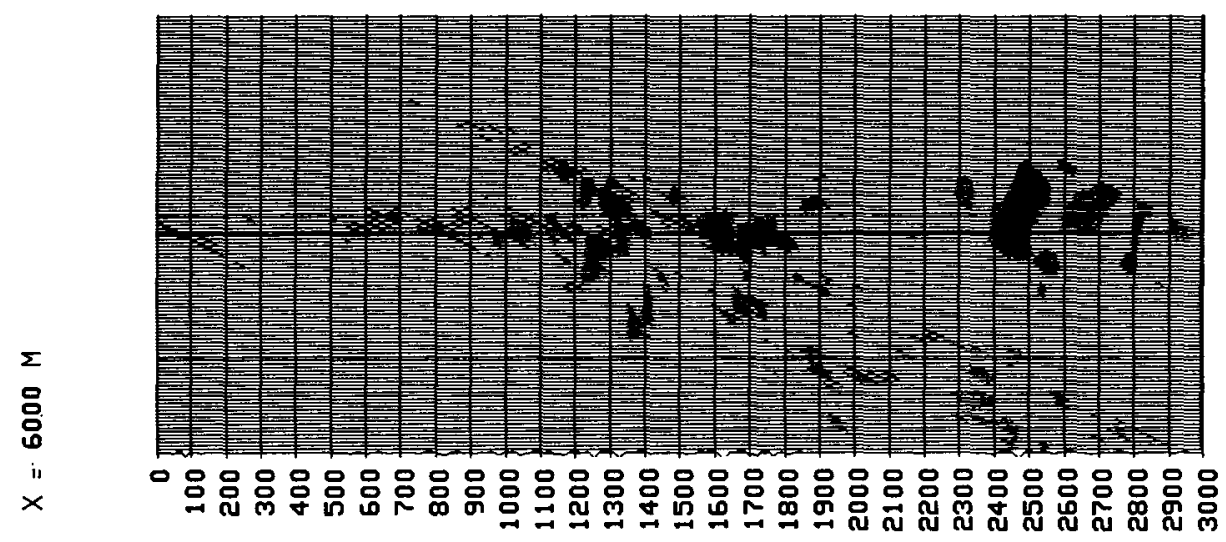

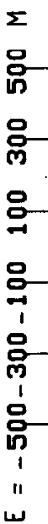

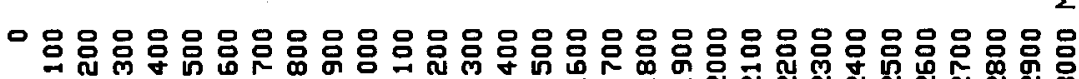

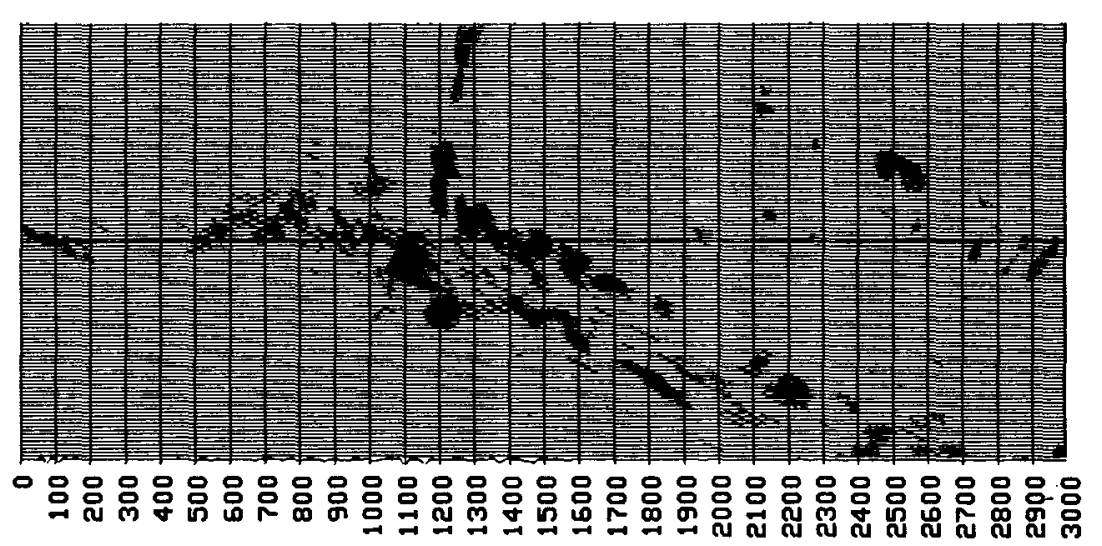

8

11

O্

검

实

E

롱욜

苋.

$\frac{\infty}{5}$

초응

สิ०

으용

눌

政

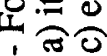

음

䅈

$\Sigma$

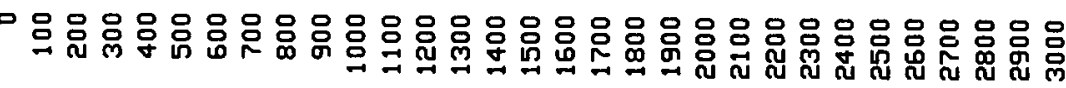

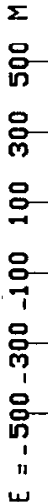

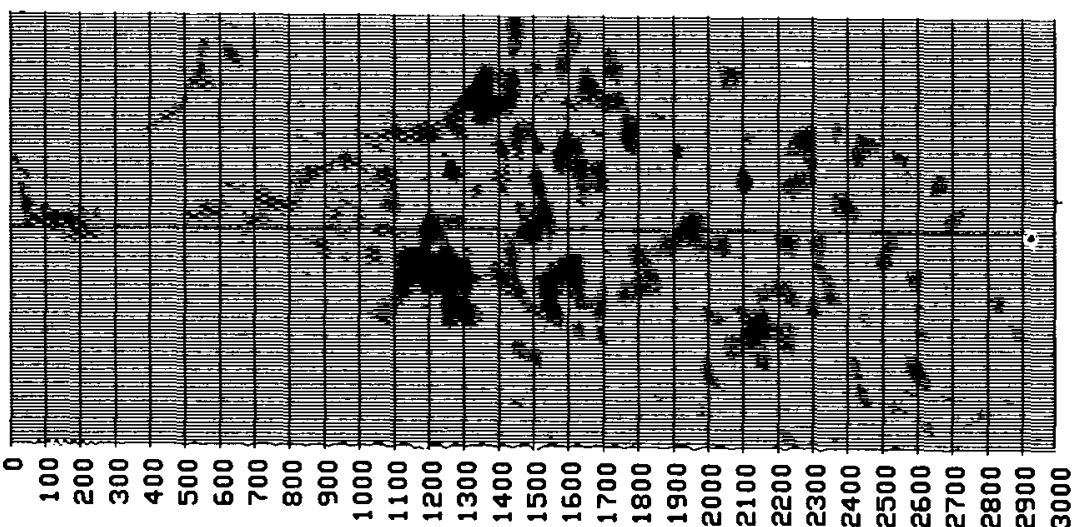




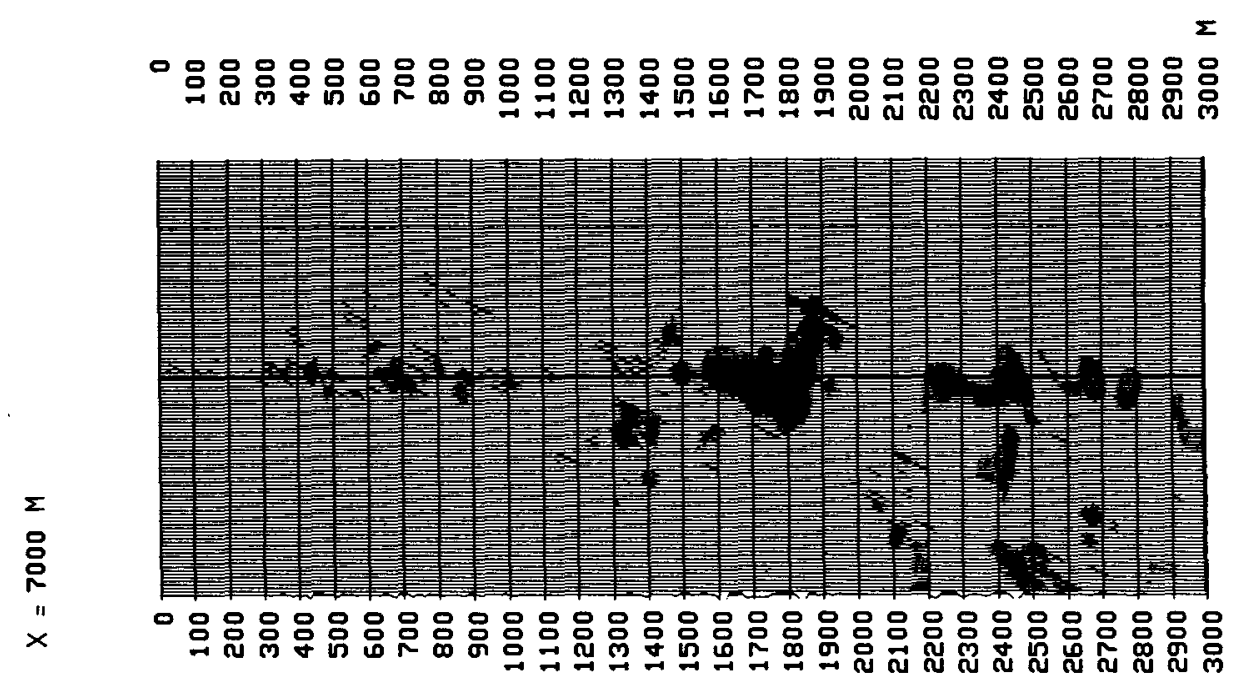

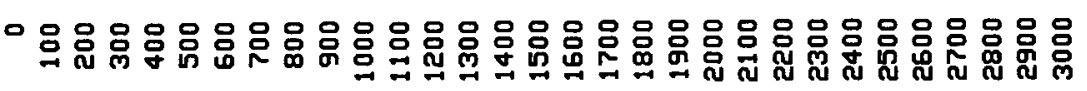

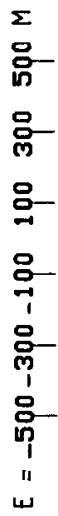

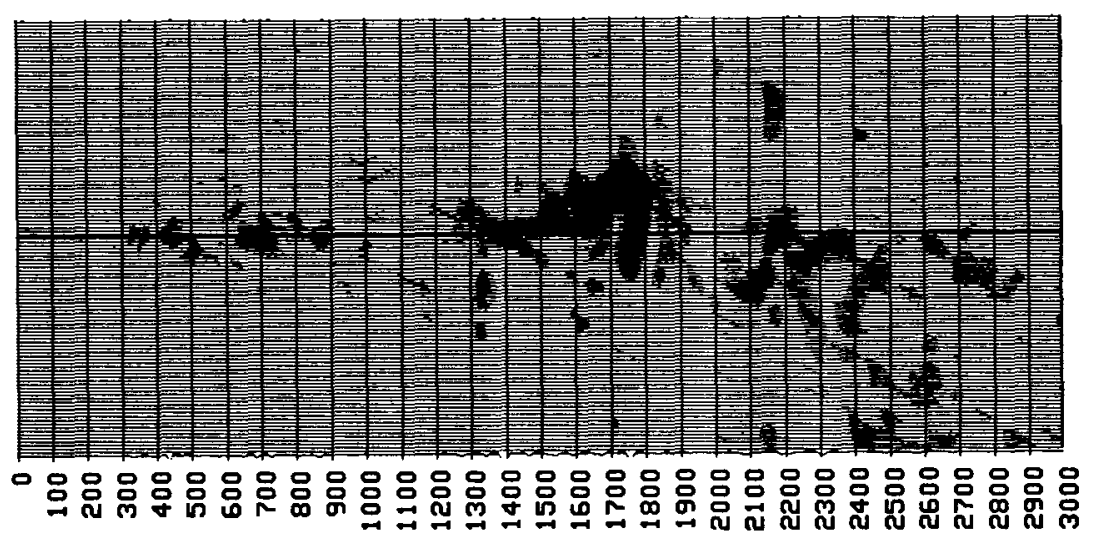

$\frac{8}{8}$

$E$
8
8
$x$

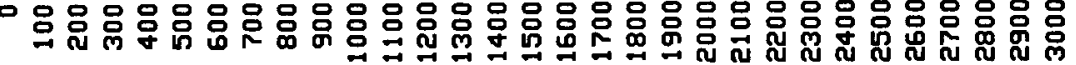

$\stackrel{x}{\infty}$

i

톨웡

की.

.

急完

สิ

60 도ㅇㅛㅛ

政

$=$

定

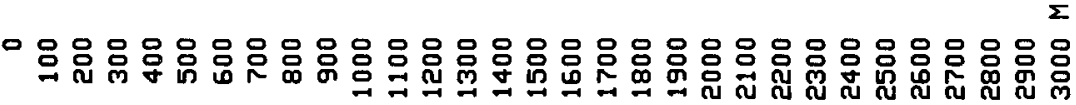

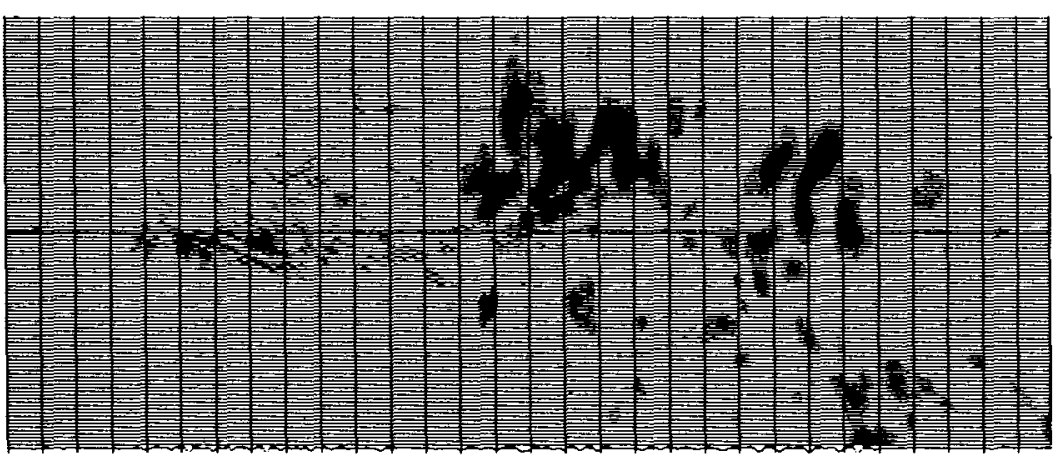

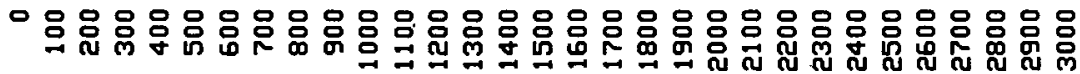


$\Sigma$

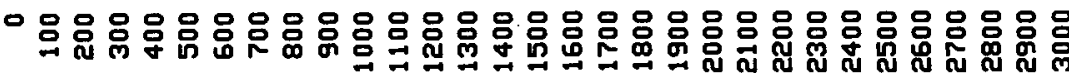

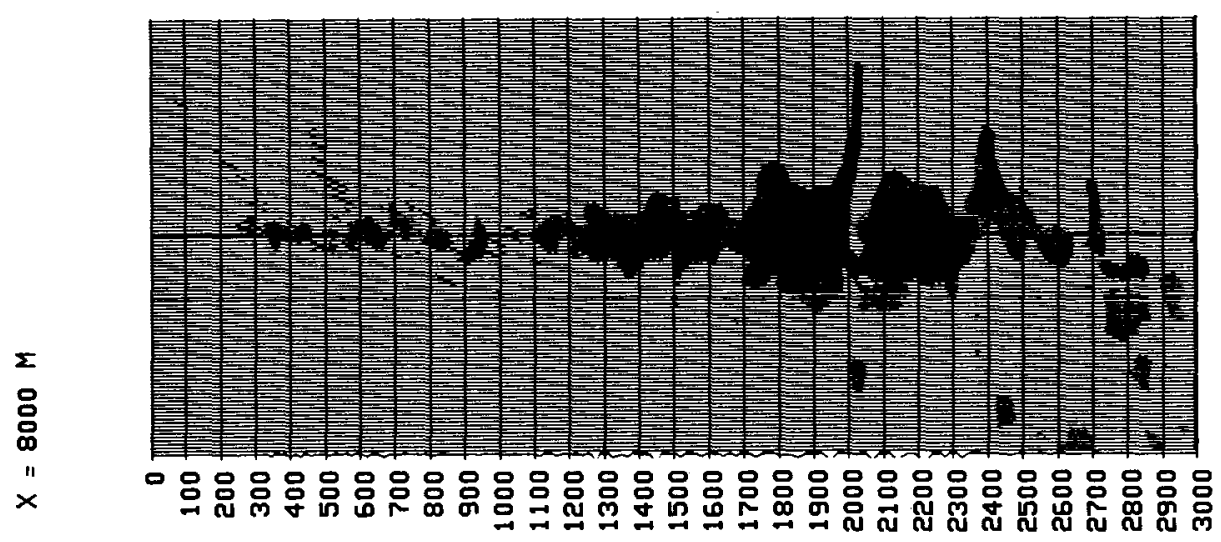

$\Sigma$

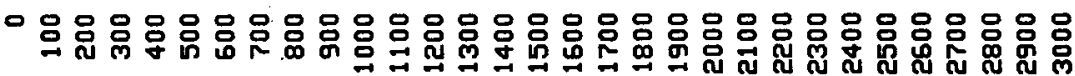
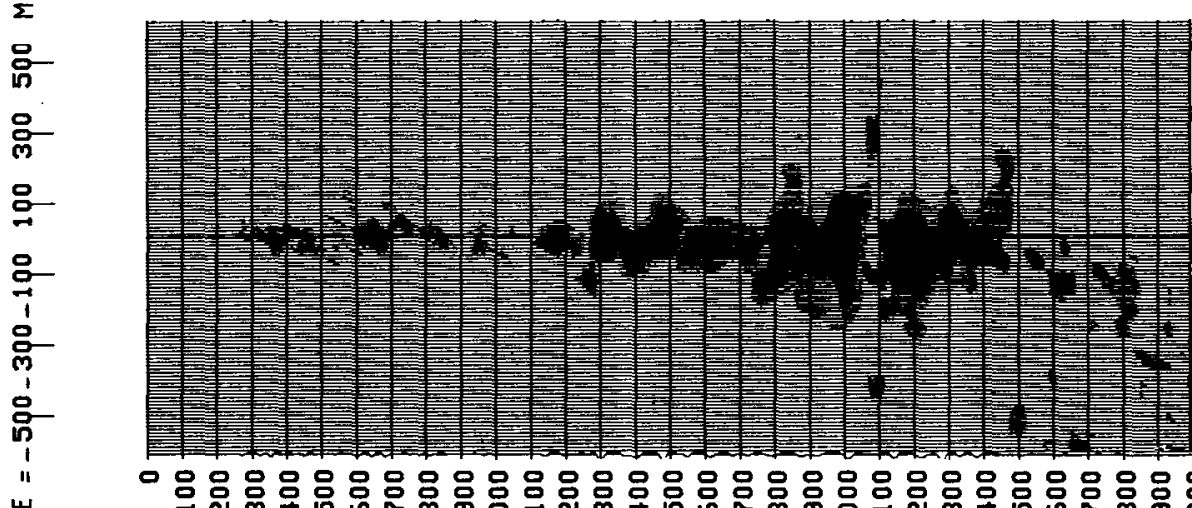

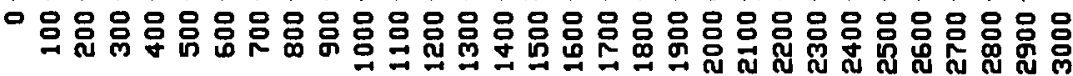

$\overbrace{8}^{8}$

กี

के

当

导 氕

응.兰

育萦

ใี่อี

요용용

承递

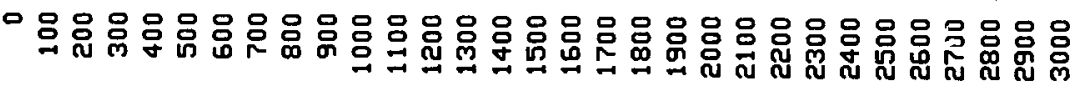

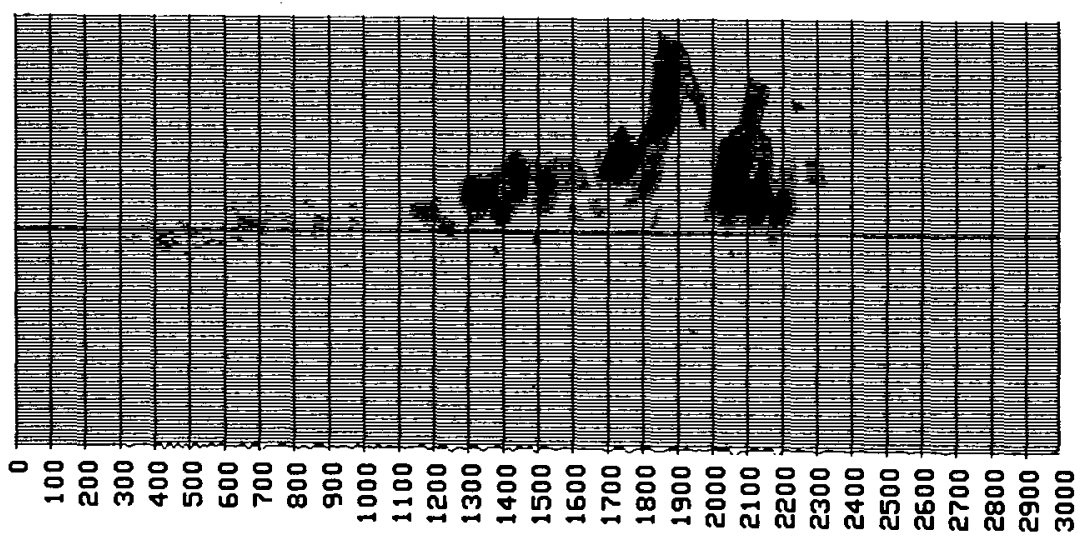

